\title{
Current Approaches in Premenstrual Syndrome Management
}

\author{
(1) Halime ABAY, id Sena KAPLAN
}

Ankara Yıldırım Beyazıt University, Department of Nursing, Ankara, Turkey

\begin{abstract}
Premenstrual syndrome (PMS) is a health problem that occurs with physical and psychological symptoms presenting about five days before menstruation, end within a few days after the onset of menstruation. The most common symptoms are appetite changes, edema, breast tenderness, abdominal pain, back pain, headache, fatigue, insomnia, depressive affect, irritability, angry outbursts andanxiety. Most women's half of lifes go through with premenstrual problems and quality of life is affected negatively. PMS prevalence is notably high in the world and in our country. In this respect, it is important to increase awareness of health professionals and women about PMS. The aim of this review is to explain current approaches of PMS management in the context of international guidelines. Getting detailed anamnesis for the diagnosis of PMS, if necessary, consultations and laboratory investigations are carried out. In addition, the diagnosis of PMS is confirmed by assessing women's symptom records for at least two months showing the type of symptoms and the time of appearance of these symptoms in the menstrual cycle. PMS management is carried out gradually by multidisciplinary team that adopted an integrative holistic approach in the direction of individualized plan. The first step includes creating awareness about PMS, teaching to women self-screening, lifestyle changes, regulation of diet, methods of coping with stress. In the second step, cognitive behavioral therapy and complementary alternative therapies are implemented. If the problem continues, there is pharmacological treatment in the third step. Finally, surgical treatment is applied in the fourth step.
\end{abstract}

Keywords: Premenstrual syndrome, disease management, women's health

\section{Introduction}

Repeated menstruation every month in women's life is considered to be a sign of being healthy. However, the symptoms that occur in the second half of the menstrual cycle negatively affect the life of most women (1). The American College of Obstetricians and Gynecologists defines the period which occurs approximately five days before menstruation and ends in a few days after menstruation starts and is accompanied by physical and psychological symptoms as premenstrual syndrome (PMS) (2). The late twenties and midfifties are the periods when PMS is seen commonly (3). In the premenstrual period, changes in appetite such as excessive eating and craving, weight gain, edema, breast tenderness, and swelling and pain in the joints, abdominal pain, stomach problems, back pain, headaches, vertigo and dizziness, sweating in hands and feet, fatigue, skin problems such as acne, insomnia and short-term drowsiness, decreased libido, depressive mood, anger outbursts, irritability, crying spells, anxiety, restlessness, and confusion have been observed $(2,3)$. Because of the problems associated with PMS, women experience change in body perception, decrease in self-confidence, social isolation and interpersonal relationships are disrupted $(4,5)$. It is also stated that PMS leads to drug addiction, increased tendency to have an accident and to commit crime, economic losses, and decline in academic achievement $(4,6)$.

The prevalence of PMS was examined, Royal College of Obstetricians and Gynaecologists reported that 4 out of 10 women have premenstrual symptoms and 5-8\% of them is severely

Address for Correspondence: Halime ABAY, Ankara Yıldırım Beyazıt University, Department of Nursing, Ankara, Turkey

Phone: +90 3129061914 E-mail: halime_colak@hotmail.com ORCID ID: orcid.org/0000-0001-9286-9755 
affected by PMS (4). In addition, PMS is seen in France at the lowest rate (12\%) and Iran at the highest rate (98\%) (7). PMS is also very common in our country and especially women in young age group are reported to have a high rate (66\%-91.8\%) of PMS in studies (8-10). Although the prevalence of PMS is influenced by cultural characteristics, PMS is a common female health problem. Given the fact that half of women's lives have passed through premenstrual period problems, it is important to raise awareness of health workers and to teach women the necessary self-care practices for PMS management. This review aims to present current approaches to PMS management accompanied by international guidelines.

\section{Etiology of PMS}

The causes of PMS are multifactorial and are still unclear $(4,11)$. Nowadays, theories suggest that there is relationship between PMS and overian hormone levels, serotonin levels, and gammaAminobucyric acid (GABA) levels. The first theories were based on the fact that PMS was associated with ovarian hormone levels. The absence of PMS before puberty, during pregnancy, after menopause, during treatment with gonanotropin relasing hormone $(\mathrm{GnRH})$ analogues suggests this situation $(4,5)$. However, there is no significant difference between symptomatic and asymptomatic women in terms of progesterone levels (5). This is explained by the theory that some women are more sensitive to progesterone (4).

One of the theories is that serotogenic activity in the brain is influenced by overial hormones. Serotonin receptors are sensitive to estrogen and progesterone. Progesteron makes an individual prone to depression by increasing monoamine oxidase which is associated with the transport of serotonin, while estrogen creates an antidepressant effect. Therefore, low estrogen levels in luteal phase and high progesterone levels in luteal phase trigger depressive mood (4).

One of the theories in the etiology of PMS is based on the relationship between progesterone and GABA. Allopregnanolone, which appears as the metabolite of progesterone, regulates the level of GABA in the blood. GABA receptors are less susceptible to allopregnanolone, since they are exposed to high concentrations of allopregnanolone prior to the luteal phase. Therefore, low concentration of allopregnanolone in luteal phase causes anxiety, depressive mood and aggression. Indeed, allopregnanolon levels were shown to be lower in women suffering from PMS $(4,5,12)$.

PMS is more likely to develop in women whose mothers have had severe PMS, and also PMS is more frequent in monozygotic twins than in dizygotic twins, suggesting that PMS has a genetic component (11). In addition to psychosocial theory, cognitive and social learning theories, and socio-cultural theory; BMI, exercise and diet are also thought to have a role in the etiology of PMS $(3,13)$.

\section{Diagnosing PMS}

Symptoms of depression, anxiety, perimenopause, chronic fatigue syndrome, irritable bowel syndrome, and thyroid disease are similar to the symptoms of PMS $(2,13)$. At the same time, half of women seeking treatment for PMS have at least one of these conditions. Depression and anxiety most often mimic the psychological symptoms of PMS. In this context, it should be determined whether the woman's mood disorder is related to the menstrual cycle (2). Therefore, women who are thought to be experiencing PMS should be consulted appropriately to rule out these diseases (5). Although there are no laboratory tests for PMS diagnosis, blood and urine analysis can be used to exclude other diseases $(3,13)$. In addition, the relationship between PMS and sexual abuse and posttraumatic stress disorder is known (14). Therefore, it is important to take detailed anamnesis of medical, reproductive and menstrual conditions in order to evaluate these aspects $(4,5)$.

The diagnosis of PMS is based on the type of symptoms and the time of emergence in the menstrual cycle (3). Premenstrual symptoms occur during the period covering the luteal phase of the endometrial cycle and ovulation phase of the ovarian cycle. The severity of symptoms increases just before the start of menstruation, and towards the end of menstruation, all symptoms are resolved. There is an asymptomatic period between the end of menstruation and the beginning of ovulation (5). In order to associate the symptoms with PMS, at least one physical and psychological symptom should occur five days before menstruation, these symptoms should end four days after menstruation, they should continue at least three menstrual cycles and should adversely affect daily activities and interpersonal relationships. It is also recommended that the structure of premenstrual symptoms be confirmed by the health worker (2). In this context, health workers should use PMS scales and prospective daily records held for at least two months $(2,4,5)$. Thus, the diagnosis made with the scale can be confirmed by diary $(5,15)$. Since women have difficulty in keeping a diary, practical mobile applications for this purpose have been developed. "PreMentricS" is one of these applications. It graphically documents premenstrual symptoms, helps healthcare workers diagnose and monitor treatment (5). These applications may be recommended for women in the young age group who are particularly concerned with technology.

\section{Management of PMS}

It is recommended that PMS Management be carried out gradually by a multidisciplinary team that has adopted an integrated holistic approach. At the same time, an individualized management plan should be applied because the number, type and severity of premenstrual symptoms vary from person to person $(4,12)$. If PSM is mild to moderate, lifestyle changes and diet causes cure and if the symptoms start to have an adverse impact on daily life pharmacological treatment is recommended (2). First step in PMS management is to create awareness with education and consultancy, to teach women self-screening and self-care practices. These are followed by non-pharmacological and pharmacological strategies and surgical methods are applied as the last option $(5,16)$ (Figure 1). 


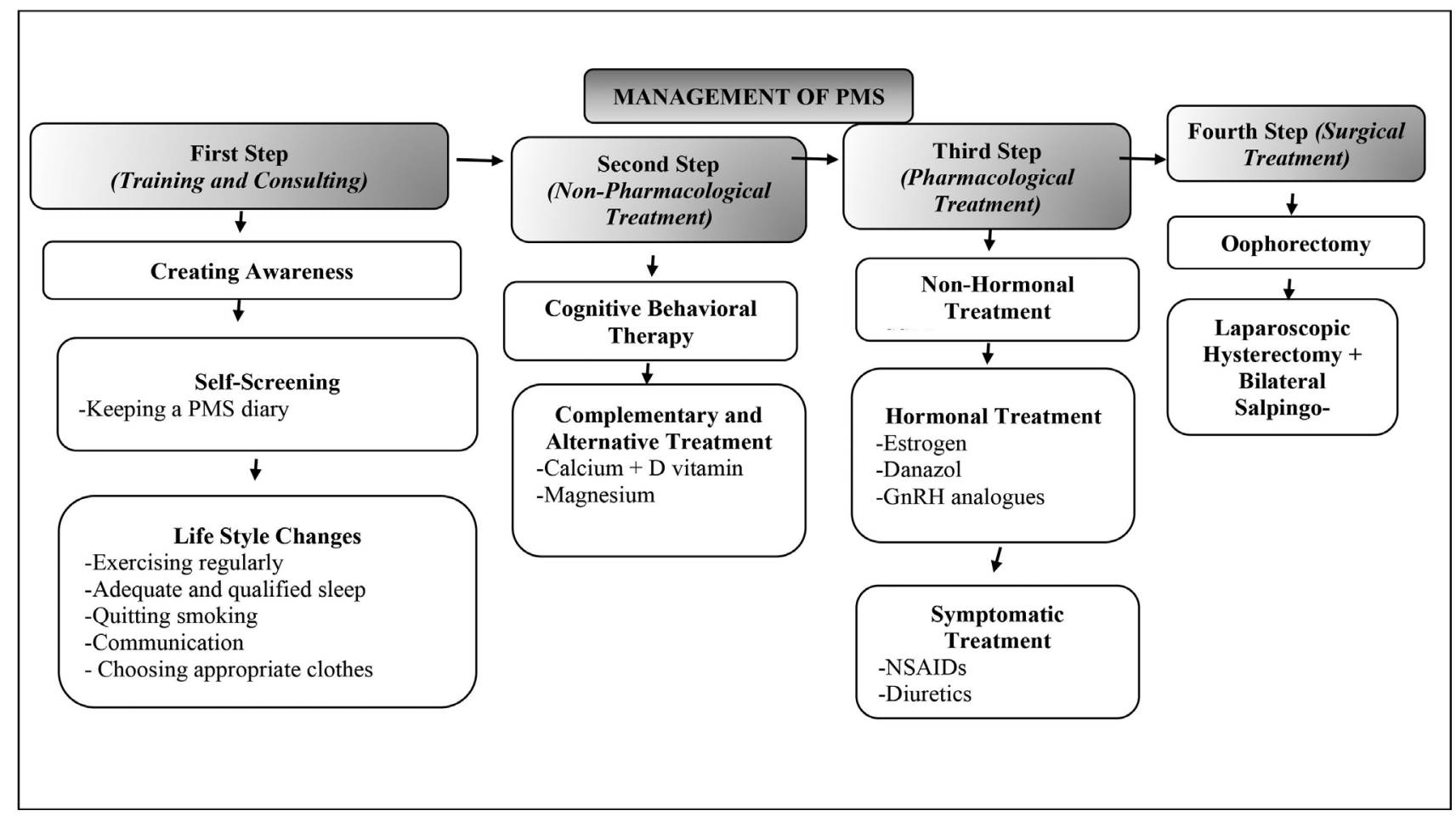

Figure 1. Premenstrual syndrome management

\section{First Step (Education and Consulting)}

\subsection{Raising Awareness}

Because most women feel premenstrual symptoms as required during the reproductive period, PMS is socially justified. For this reason, the vast majority of women do not seek treatment for PMS. Although this situation varies according to cultures, women in our country do not think that PMS can be treated and do not refer to the health care institution because they hesitate to talk to others about it (17). Therefore, health workers are advised to evaluate all women admitted to health institution during the reproductive period in terms of PMS, to organize trainings to make them aware of PMS management and to make them consulting (12).

\subsection{Self-Screening}

Women diagnosed with PMS should be informed about the events occurring on ovaries and uterus every month in order to create awareness of menstrual cycle. It should be explained that most women have PMS and can manage PMS. So the woman who has the awareness of PMS and the belief that PMS can be managed will ask the question "How can I help myself?". In this case, firstly, it is suggested that the woman should keep a PMS diary so that she can recognize the symptoms she has experienced and determine the type and severity of symptoms, when and how they occur, and how much they affect her life. For example, women who hold a diary can rearrange their meetings that correspond to the premenstrual period and that have the potential to pass through difficult, and begin to work early on their exams (17).

\subsection{Lifestyle Changes}

Most women can only cope with PMS by making lifestyle changes (18). In this context, it is important that health workers guide women. As lifestyle changes in the management of PMS;

-Women are advised to exercise at least 30 minutes a day. Aerobic exercises, including walking, running, cycling, and swimming increases heart rate, lung function, sleep quality and endorphin levels, while reducing fatigue and depressive mood $(2,3,19,20)$.

-Adequate (at least 8 hours a day) and qualified sleep is recommended to reduce fatigue and depressive mood (3).

-Smoking is recommended to be stopped, because it is known that nicotine worsens premenstrual symptoms (3).

-It is recommended that women communicate with their spouses, family members and friends and share their feelings in order to be understood about how they feel during this period and to receive help from social support systems $(3,13,21)$.

-If there is a general edema in the body, clothing which is not tight should be preferred; if edema is in feet, comfortable and supportive shoes should be worn; and if edema is in breasts, supportive bras and elastic waist band are recommended $(3,22)$. 


\subsection{Diet Regulation}

It is important to note that the prevalence of PMS in women with high BMI is higher and diet regulation is important in management of PMS (23). In this context, nutrition should be modified as follows;

-Six small meals, rather than three main meals per day should be preferred $(2,3)$,

-Less fat, sugar, alcohol consumption and more fiber, vegetables and fruit consumption are recommended $(2,3)$,

-To reduce irritability and insomnia caffeine (tea, coffee, cola) intake and to reduce edema, salt consumption should be reduced and natural diuretics such as grapefruit to should be added to $\operatorname{diet}(2,3,20)$,

-The consumption of whole grain bread, barley, brown rice, beans and lentils should be encouraged, as well as feeding with nutrients which contain high level of protein, pulp and complex carbohydrates can reduce psychological symptoms and craving (2),

-Iron-rich foods should be consumed with sources of vitamin C to replace iron lost in menstruation,

-Walnuts, chia seeds and flax seeds (1 spoonful/day) which are rich of omega-3 and fatty fishes should be added to the diet (24),

-Consuming calcium-rich foods such as yoghurt and green leafy vegetables is recommended (2),

-Healthy snacks (whole grain cereals, dried fruits, nuts, oily seeds) is recommended to be preferred $(12,22)$.

\subsection{Coping With Stress}

Because stress increases the severity of PMS, coping with stress significantly simplifies PMS management (3). In this context, the recommended methods of coping with stress are;

-Breathing exercises,

-Relaxation exercises such as meditation and yoga,

-Having a bath, sleeping enough, dealing with a hobby, massage, biofeedback, autohypnoses and acupressure $(22,25)$.

\section{Second Step (Non-Pharmacological Treatment)}

If daily life, work life or interpersonal relationships are negatively affected, then a woman is in search of PMS's treatment. In this case, non-pharmacological treatment in the second step is recommended to women. Non-pharmacological treatment methods include cognitive behavioural therapy (CBT) and complementary and alternative treatment (CAT) (3).

\subsection{Cognitive Behavioral Therapy}

In the management of PMS, with CBT which is a routine treatment option, the woman's cultural perception of PMS and negative menstrual attitudes are changed $(3,4,15,22,26,27)$. At the same time, relaxation, stress management and assertiveness are provided with CBT. With a successful CBT, drug treatment can be avoided $(3,4)$. Although the studies show that CBT do not provide rapid improvement in the management of PMS as well as antidepressant treatment, it is at least as effective as antidepressant treatment and has a longer lasting effect $(5,12)$.

\subsection{Complementary and Alternative Treatment}

Although some CAT practices are known to be useful in the management of PMS, there is no advanced evidence that the majority of them are effective. In this regard, women should be advised to consult their physician before applying CAT. The physician should consider two points in CAT practices: The first is that the data obtained from clinical studies on CAT practices are limited and not strong. Second, if the individual has drugs to use, it is possible that drugs may interact with the CAT practices $(2-5)$.

The most convincing of the recommended CAT practices in the management of PMS is calcium + vitamin D $(2,4)$. Studies show that blood calcium and vitamin D levels are lower in women who suffer from PMS (4,12). Daily average $1000 \mathrm{mg}$ calcium supplements are recommended to improve nearly all symptoms $(15,16)$.

Another recommended CAT practice in the management of PMS is magnesium supplementation $(2,4)$. Daily $400 \mathrm{~g}$ magnesium intake improves premenstrual symptoms associated with depressive mood and fluid retention. The combination of magnesium $+\mathrm{B} 6$ is also recommended in the management of PMS $(16,28)$. In addition, there are studies showing that vitamin E, vitex agnus castus, saffron, ginkgo biloba, evening primrose oil, lemon grass (lemon balm), curcumin, wheat seed, isoflavones, St Jhon's Wort oil, multivitamins, refloxology and acupuncture are effective in the management of PMS, but there is insufficient evidence $(2-5,12,13,16,20,22,29,30)$.

\section{Third Stage (Pharmacological Treatment)}

In PMS, there are treatment options suitable for etiological theories. The first option is non-hormonal therapy to increase the central seratogenic transmission, and the second option is hormonal therapy to increase the ovarian hormone level. At the same time, symptomatic treatment may be applied to improve some premenstrual symptoms (11).

\subsection{Non-Hormonal Treatment}

SSRIs are the first choice in non-hormonal therapies and their use increases serotonin activity in the brain to improve PMS $(4,12,15,31)$. Indeed, studies show that SSRIs are effective in PMS management compared to placebo $(5,16,31,32)$. However, some of the drugs in this group may have quite a lot of side effects (4). In this regard, women should be informed about the possible side effects of SSRIs such as nausea, insomnia, drowsiness, fatigue and decreased libido in continuous use $(4,5)$. In order to avoid withdrawal symptoms, women should be told to stop taking the medication gradually. In addition, intermittent use of SSRIs may be recommended for the reduction of side 
effects (4). While intermittent use of SSRIs is as effective as continuous use in reducing psychological symptoms, there is no similar situation in reducing physical symptoms (5). If SSRIs are to be used intermittently, they should be started before PMS occurs and continued throughout the luteal phase (4.5). In addition, if anxiety is a major problem among premenstrual symptoms and SSRIs can not solve this problem, an anti-anxiety drug is recommended (4). In addition, women receiving SSRIs should be advised that SSRIs are not appropriate for use during pregnancy (4).

\subsection{Hormonal Treatment}

Hormonal therapy can improve physical symptoms by suppressing ovulation and reducing hormonal fluctuations, while psychological symptoms can not be treated $(3,4)$. Estrogen, danazol and $\mathrm{GnRH}$ are used in hormonal therapy.

-Estrogen: Oral contraceptives containing drospirenone are the first choice among hormonal interventions $(4,33)$. It is reported that the use of hormone pills for 24 days and inactive pills for 4 days has improved significantly in PMS compared to placebo $(5,15,33)$. Estrogen can be used as a transdermal patch and subcutaneous implant. However, oral contraceptives are not suitable for every woman as they pose a risk of venous thromboembolism. At the same time, if the estrogen is not met with progesterone, it causes hyperplasia in the breast and endometrium tissue, and the data on the safe dose of estrogen is insufficient. Since synthetic progestestin causes PMS-like symptoms, it should be given in minimal doses (the dose should not exceed the maximum amount produced from corpus luteum). Alternatively, progesterone can be applied directly to the endometrial layer with an intrauterine device containing levonorgestrel $(4,5)$. If pregnancy is not desired, the woman should be informed about the use of a barrier method and not rely on estrogen to suppress ovulation (4). In addition, studies have shown that the use of older generation combined oral contraceptives and progesterone alone are not useful in the treatment of PMS $(4,5,12,34)$.

-Danazol: Low-dose danazol therapy at luteal phase is effective in reducing the sensitivity of the breasts. However, the potential for irreversible virilism should be taken into consideration. Because danazol may cause virilism also in the fetus, the woman should be told about the importance of using a reliable contraceptive method in this process (4).

-GnRH: GnRH analogues are highly effective in the treatment of severe PMS and are not recommended for use in routine. If there is a severe PMS that requires effective and long-term treatment, then GnRH should be preferred. Hot flashes, night sweats, insomnia and depressive mood are the short-term effects and vaginal atrophy, increased cardiovascular disease and osteoporosis risk are the long-term effects of hypoestrogenic condition. Therefore, GnRH analogues should not be used for longer than six months and if re-use is planned, bone mineral density should be evaluated prior to use it again $(4,5,16)$.

\subsection{Symptomatic Treatment}

-Nonsteroidal Anti-inflammatory Drugs (NSAIDs): Analgesic and anti-inflammatory effects of NSAIDs that inhibit prostaglandin synthesis are used to improve PMS. NSAIDs can help reduce cramps, headaches, back pain, sensitivity in the breasts. However, women should be informed about the longterm use of these drugs, which may lead to stomach bleeding or ulcers $(2,3)$.

-Diuretics: Diuretics used to treat severe premenstrual symptoms associated with fluid retention may cause undesirable effects when taken together with some drugs. For example, concomitant use of diuretics with NSAIDs may cause kidney problems. In this respect, the woman should be questioned whether she is taking another drug before the diuretic is recommended $(2,4)$.

\section{Fourth Stage (Surgical Treatment)}

\subsection{Oophorectomy}

Oophorectomy alone removes PMS, but estrogen replacement is needed after surgery. In this case, preventive progesterone therapy is needed to prevent endometrial hyperplasia (5).

\subsection{Laparoscopic Hysterectomy + Bilateral Salpingo- oophorectomy}

Prevention of ovulation by performing hysterectomy with bilateral salpingo-oophorectomy is highly successful in the treatment of severe PMS $(3,4)$. However, since hysterectomy + bilateral salpingo-oophorectomy is a serious treatment method, it should only be preferred if pharmacological treatment fails, long-term use of $\mathrm{GnRH}$ analogues is required or there is a different gynecological condition requiring surgery (4). This treatment method should be decided considering the anesthetic risks, surgical complications, infertility and the development of surgical menopause. If a hysterectomy + bilateral salpingooophorectomy is to be performed, it is recommended to be performed laparoscopically, because it is less invasive. Hormone replacement therapy is recommended for women under 45 years of age after the surgery $(4,5)$. In addition, studies have shown that hysterectomy alone or endometrial ablation is not sufficient in the treatment of PMS $(5,16)$.Prevention of ovulation by performing hysterectomy with bilateral salpingo-oophorectomy is highly successful in the treatment of severe PMS $(3,4)$. However, since hysterectomy + bilateral salpingo-oophorectomy is a serious treatment method, it should only be preferred if pharmacological treatment fails, long-term use of $\mathrm{GnRH}$ analogues is required or there is a different gynecological condition requiring surgery (4).

\section{Conclusion}

PMS is a common health problem, and women spend half their life with premenstrual problems and experience a reduction in self-confidence, social isolation, decline in academic achievement, increase in accident tendency and loss of Labor. With the management of PMS, the quality of life can be improved. The first step in PMS management is to create awareness, to make women self-screen, adopt lifestyle changes, apply nutrition 
suggestions and use stress coping methods. If these methods are insufficient, CBT and CAT in the second step are applied. If PMS continues to affect daily life negatively; hormonal, non-hormonal and symptomatic treatment in the third step; oophorectomy or laparoscopic hysterectomy + bilateral salpingooopherectomy in the fourth step are applied. In this context, it is important for women suffering from PMS to be monitored by health workers and it is recommended to establish our national PMS management guide and to develop models in PMS management and to increase evidence-based research. At the same time, it is recommended that women who are admitted to primary health care institutions should be evaluated routinely in terms of PMS. Also it is recommended to give awareness training for young people in high schools and universities, to set up counseling units, and to organize social awareness campaigns using the press organs.

Peer Review: Externally peer-reviewed.

\section{Authorship Contributions}

Concept: H.A., S.K., Design: H.A., S.K., Data Collection or Processing: - Analysis or Interpretation: H.A., S.K., Literature Search: H.A., Writing: H.A.

Conflict of Interest: No conflict of interest was declared by the authors.

Financial Disclosure: The authors declared that this study received no financial support.

\section{References}

1. İşik H, Ergöl Ş, Aynioğlu Ö, Şahbaz A, Kuzu A, Uzun M. Premenstrual syndrome and life quality in Turkish health science students. Turk J Med Sci 2016;46:695-701.

2. The American College Of Obstetricians And Gynecologists. 2015. Premenstrual syndrome (PMS). Available from: URL: $\quad$ https://www.acog.org/-/media/For-Patients/faq057. pdf?dmc=1\&ts=20171211T1813370296 (11.12.2017)

3. Women's Health Concern. 2012. Premenstrual syndrome (PMS). Available from: URL: https://www.womens-health-concern. org/_wpress/wp-content/uploads/2015/02/WHC_FS_PMS.pdf (11.12.2017)

4. Green LJ, O'Brien PMS, Panay N, Craig M; on behalf of the Royal College of Obstetricians and Gynaecologists. Management of premenstrual syndrome. BJOG 2017;124:73-105.

5. Walsh S, Ismaili E, Naheed B, O’Brien S. Diagnosis, pathophysiology and management of premenstrual syndrome. The Obstetrician Gynaecologist 2015;17:99-104.

6. Tolossa FW, Bekele ML. Prevalence, impacts and medical managements of premenstrual syndrome among female students: cross-sectional study in college of health sciences, Mekelle University, Mekelle, Northern Ethiopia. BMC Womens Health 2014;29:52-61.

7. A DM, K S, A D, Sattar K. Epidemiology of premenstrual syndrome (PMS)-a systematic review and meta-analysis study. J Clin Diagn Res 2014;8:106-9.
8. Kahyaoglu Sut H, Mestogullari E. Effect of premenstrual syndrome on work-related quality of life in Turkish nurses. Saf Health Work 2016;7:78-82.

9. Goker A, Artunc-Ulkumen B, Aktenk F, Ikiz N. Premenstrual syndrome in Turkish medical students and their quality of life. J Obstet Gynaecol 2015;35:275-8.

10. Alpaslan AH, Avci K, Soylu N, Tas HU. Association between premenstrual syndrome and alexithymia among Turkish University students. Gynecol Endocrinol 2014;30:377-80.

11. European Medicines Agency, 2011. Guideline on the treatment of premenstrual dysphoric disorder (PMDD). Available from: URL: http://www.ema.europa.eu/docs/en_GB/document_library/ Scientific_guideline/2011/08/WC500110103.pdf (11.12.2017)

12. Panay N. Management of premenstrual syndrome: evidence-based guidelines. Obstet Gynaecol Reprod Med 2011;21:221-8.

13. Khajehei M. Aetiology, diagnosis and management of premenstrual syndrome. J Pain Relief 2015;4:1-4.

14. Bertone-Johnson ER, Whitcomb BW, Missmer SA, Manson JE, Hankinson SE, Rich-Edwards JW. Early Life Emotional, Physical, and Sexual Abuse and the Development of Premenstrual Syndrome: A Longitudinal Study. J Womens Health (Larchmt) 2014;23:729-39.

15. Hofmeister S, Bodden S. Premenstrual syndrome and premenstrual dysphoric disorder. Am Fam Physician 2016;94:236-40.

16. Daugherty JE. Treatment Strategies for Premenstrual Syndrome. Am Fam Physician 2017;58:183-92.

17. Öztürk S, Tanrıverdi D. Premenstrual sendrom ve başetme. Anadolu Hemşirelik ve Sağlık Bilimleri Dergisi 2010;13:57-61.

18. Mohebbi M, Akbari SAA, Mahmodi Z, Nasiri M. Comparison between the lifestyles of university students with and without premenstrual syndromes. Electron Physician 2017;9:4489-96.

19. Tsai SY, Kuo FC, Kuo HC, Liao LL. The prevalence of self-reported premenstrual symptoms and evaluation of regular exercise with premenstrual symptoms among female employees in Taiwan. Women Health 2017;20:1-13.

20. Maharaj S, Trevino K. A Comprehensive Review of Treatment Options for Premenstrual Syndrome and Premenstrual Dysphoric Disorder. J Psychiatr Pract 2015;21:334-50.

21. Rezaee H, Mahamed F, Amidi Mazaheri M. Does Spousal Support Can Decrease Women's Premenstrual Syndrome Symptoms? Glob J Health Sci 2016;8:19-26.

22. Kelderhouse K, Taylor JS. A review of treatment and management modalities for premenstrual dysphoric disorder. Nurs Womens Health 2013;17:294-305.

23. Haghighi ES, Jahromi MK, Daryano Osh F. Relationship between cardiorespiratory fitness, habitual physical activity, body mass index and premenstrual symptoms in collegiate students. J Sports Med Phys Fitness 2015;55:663-7.

24. Behboudi-Gandevani S, Hariri FZ, Moghaddam-Banaem L. The effect of omega 3 fatty acid supplementation on premenstrual syndrome and health-related quality of life: a randomized clinical trial. J Psychosom Obstet Gynaecol 2017;14:1-7.

25. Bazarganipour F, Taghavi SA, Allan H, Beheshti F, Khalili A, Miri F, et al. The effect of applying pressure to the LIV3 and LI4 on the 
symptoms of premenstrual syndrome: A randomized clinical trial. Complement Ther Med 2017;31:65-70.

26. Ussher JM, Perz J. Evaluation of the relative efficacy of a couple cognitive-behaviour therapy (CBT) for Premenstrual Disorders (PMDs), in comparison to one-to-one CBT and a wait list control: A randomized controlled trial. PLoS One 2017;12:1-25.

27. Izadi-Mazidi M, Davoudi I, Mehrabizadeh M. Effect of group cognitive-behavioral therapy on health-related quality of life in females with premenstrual syndrome. Iran J Psychiatry Behav Sci 2016;10:2-5.

28. Fathizadeh N, Ebrahimi E, Valiani M, Tavakoli N, Yar MH. Evaluating the effect of magnesium and magnesium plus vitamin B6 supplement on the severity of premenstrual syndrome. Iran J Nurs Midwifery Res 2010;15(Suppl1):401-5.

29. Hausenblas HA, Heekin K, Mutchie HL, Anton S. A systematic review of randomized controlled trials examining the effectiveness of saffron (Crocus sativus L.) on psychological and behavioral outcomes. J Integr Med 2015;13:231-40.

30. Jang SH, Kim DI, Choi MS. Effects and treatment methods of acupuncture and herbal medicine for premenstrual syndrome/ premenstrual dysphoric disorder: systematic review. BMC Complement Altern Med 2014;14:1-13.

31. Marjoribanks J, Brown J, O’Brien PMS, Wyatt K. Selective serotonin reuptake inhibitors for premenstrual syndrome. Cochrane Database Syst Rev 2013;6:1-159.

32. Reid RL, Soares CN. Premenstrual Dysphoric Disorder: Contemporary Diagnosis and Management. J Obstet Gynaecol Can 2017;2163:30524-8.

33. Lopez LM, Kaptein AA, Helmerhorst FM. Oral contraceptives containing drospirenone for premenstrual syndrome. Cochrane Database Syst Rev 2012;2:CD006586.

34. Ford O, Lethaby A, Roberts H, Mol BW. Progesterone for premenstrual syndrome. Cochrane Database Syst Rev 2012;3:CD003415. 